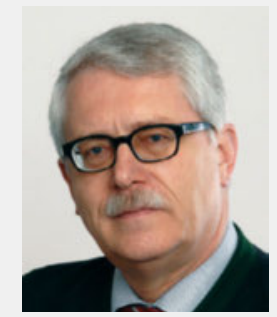

Prof. Dr. Günter Schmolz

\title{
Tropenkrankheiten und mehr!
}

Liebe Kolleginnen und Kollegen,

in der ersten Kasuistik beschreiben Veronika Lermer und Jörg Schneider eine Kältepannikulitis bei einer 51 -jährigen Skifahrerin, zugezogen bei $-18^{\circ} \mathrm{C}$ in den Chiemgauer Alpen. Die Fallbeschreibung dieses seltenen Krankheitsbilds wird durch eine Klassifikation der Pannikulitiden ergänzt.

Louise Roggelin et al. stellen einen dramatischen Fall einer Melioidose bei einem 69-Jährigen vor, der sich in Thailand mit $42{ }^{\circ} \mathrm{C}$ Fieber und einem akuten Nierenversagen in einer klinischen Notfallambulanz vorstellte und dann in Deutschland weiterbehandelt wurde. Diese, durch Burkholderia pseudomallei hervorgerufene bakterielle Infektion, ist in weiten Teilen der Welt verbreitet, vor allem in Südostasien und Nordaustralien. Nach Deutschland importierte Fälle sind zwar sehr selten, nach Ansicht des RKI aber unterdiagnostiziert, zumal keine Meldepflicht besteht. B.-pseudomallei-Infektionen sind potenziell lebensbedrohlich, die Letalität liegt bei bis zu $50 \%$. Das CDC hat den Erreger in die Kategorie B möglicher Biowaffen eingeordnet. Die Anzüchtung von B. pseudomallei setzt ein Labor mindestens der Sicherheitsstufe BSL 3 voraus.

Die Versorgung medizinischer Notfälle auf Offshorewindkraftanlagen unterliegt speziellen Anforderungen, die Bedingungen sind schwierig: kleine Teams, beengte Verhältnisse, gefährliche Bergung. In den letzten Jahren konnte mit der Einbindung von Telenotärzten eine deutliche Verbesserung des Hilfs- und Rettungsmanagements erreicht werden. Marco Loddo stellt die Situation, die erforderliche medizinische Kompetenz der Einsatzkräfte und den rechtlichen Rahmen dar und bereichert seinen Beitrag mit Kasuistiken.

Hinrich Sudeck richtet unseren Blick auf vernachlässigte Tropen- und Armutskrankheiten in Südeuropa. Er macht deutlich, dass wir uns auf neu importierte und selten vorkommende Infektionskrankheiten einstellen müssen. Das Spektrum reicht vom Denguefieber über die Leishmaniose bis zur Strongyloidosis. Keineswegs ausgemacht ist die Begrenzung auf Dauer von Vektoren und Erregern in dieser Region. Das gefährliche und von Mensch zu Mensch übertragbare Krim-Kongo-Fieber (CCHF) ist zwar derzeit in Deutschland nicht bekannt, war aber möglicherweise bereits in der Eisenzeit hier endemisch, wie Befunde in Grabbeigaben eines keltischen Fürsten bei der Heuneburg in Baden-Württemberg zeigen (Wiktorowicz CJ et al. Journal of Archaelogical Science 2017; 78: 29-39).

Den Schluss bildet ein Beitrag von Winfried Maaßen et al. über die Erfahrungen von Tropenärzten der Bundeswehr in Mali. Die Lektüre ist für jeden Schwarzafrikafahrer ein Muss, für alle anderen unverzichtbar.

Ich wünsche Ihnen eine anregende oder gar aufregende Lektüre. 